\title{
Clofazimine, but Not Isoniazid or Rifampicin, Augments Platelet Activation in vitro
}

\author{
Ronald Anderson ${ }^{1,2 *}$, Annette J. Theron ${ }^{1}$, Jan G. Ne/3,4, Chrisna Durandt ${ }^{1,2}$, \\ Moloko C. Cholo ${ }^{1}$, Charles Feldman ${ }^{5}$ and Gregory R. Tintinger ${ }^{6}$
}

1 Department of Immunology, Faculty of Health Sciences, University of Pretoria, Pretoria, South Africa, ${ }^{2}$ Institute for Cellular and Molecular Medicine, Faculty of Health Sciences, University of Pretoria, Pretoria, South Africa, ${ }^{3}$ Department of Haematology, Faculty of Health Sciences, University of Pretoria, Pretoria, South Africa, ${ }^{4}$ Tshwane Academic Division, National Health Laboratory Service, Pretoria, South Africa, ${ }^{5}$ Department of Internal Medicine, Faculty of Health Sciences, University of the Witwatersrand, Johannesburg, South Africa, ${ }^{6}$ Department of Internal Medicine, Faculty of Health Sciences, Steve Biko Academic Hospital, University of Pretoria, Pretoria, South Africa

\section{OPEN ACCESS}

Edited by:

Mohamed M. Abdel-Daim,

Suez Canal University, Egypt

Reviewed by:

Marta Chagas Monteiro, Universidade Federal do Pará, Brazil

Essa M. Saied,

Humboldt-Universität zu Berlin,

Germany

Mohamed Elbadawy,

Benha University, Egypt

*Correspondence:

Ronald Anderson

ronald.anderson@up.ac.za

Specialty section:

This article was submitted to Inflammation Pharmacology,

a section of the journal

Frontiers in Pharmacology

Received: 25 September 2018

Accepted: 30 October 2018

Published: 20 November 2018

Citation:

Anderson R, Theron AJ, Nel JG,

Durandt C, Cholo MC, Feldman C and Tintinger GR (2018) Clofazimine, but Not Isoniazid or Rifampicin, Augments Platelet Activation in vitro.

Front. Pharmacol. 9:1335.

doi: 10.3389/fphar.2018.01335
Although the inclusion of the cationic amphiphilic, anti-mycobacterial agent, clofazimine, in the chemotherapeutic regimens of patients with multidrug-resistant tuberculosis (TB) has contributed to improved outcomes, concerns remain about the cardiotoxic potential of this agent. Accordingly, the current study was undertaken with the primary objective of investigating the effects of clofazimine, on the reactivity of human platelets in vitro, a seemingly unexplored, mechanism of cardiotoxicity. Platelet-rich plasma (PRP) prepared from the blood of healthy, adult humans was treated with clofazimine $(0.625-$ $10 \mathrm{mg} / \mathrm{L}$ ), or the primary anti-TB agents, isoniazid and rifampicin (at final concentrations of 5 and $10 \mathrm{mg} / \mathrm{L}$ ), followed by addition of either adenosine $5^{\prime}$-diphosphate (ADP) or thrombin and measurement of platelet activation according to the magnitude of expression of CD62P (P-selectin), as well as the CD62P-mediated formation of heterotypic neutrophil:platelet (NP) aggregates, using flow cytometry. Clofazimine, but neither isoniazid nor rifampicin, caused dose-related potentiation of both ADP- and thrombin-activated expression of CD62P by platelets, achieving statistical significance at threshold concentrations of 0.625 and $2.5 \mathrm{mg} / \mathrm{L}$, respectively, as well as significant formation of $\mathrm{N}: \mathrm{P}$ aggregates. These stimulatory effects of clofazimine on platelet activation were partly attenuated by pre-treatment of PRP with the membrane-stabilizing agent, $\alpha$-tocopherol, possibly consistent with a membrane-disruptive mechanism. In conclusion, clofazimine, at concentrations within the therapeutic range, augments platelet activation in vitro, probably by a mechanism linked to membrane destabilization. If operative in vivo, these pro-thrombotic activities of clofazimine may predispose for development of microvascular occlusion, exacerbating an already existing high risk for development of TB-associated cardiovascular disease.

Keywords: adenosine $5^{\prime}$-diphosphate, clofazimine, isoniazid, neutrophils, platelets, P-selectin, rifampicin, thrombin 


\section{INTRODUCTION}

Recent developments in the antimicrobial chemotherapy of multidrug-resistant and extensively drug-resistant (MDR/XDR)tuberculosis (TB) include recognition of the therapeutic efficacy of the "repurposed" riminophenazine agent, clofazimine (Aung et al., 2014; Tang et al., 2015; Trébucq et al., 2018) which appears to target both replicating and non-replicating Mycobacterium tuberculosis bacilli (Cholo et al., 2017). Concerns remain, however, about the risk for development of cardiac dysfunction during chemotherapy with this agent, specifically prolongation of the cardiac QT interval, an indicator of development of ventricular tachyarrhythmias and possibly cardiac arrest (Choudhri et al., 1995; Li et al., 2016; Wallis, 2016). The risk of drug-related cardiotoxicity in patients with TB may be further exacerbated by the fact that the infection per se is associated with systemic inflammation and a pro-thrombotic state, characterized by an increased risk of acute coronary events (Chung et al., 2014; Huaman et al., 2017). This risk may be intensified firstly by prolonged disease and extended chemotherapy, particularly in those patients with comorbid disease (Pepper et al., 2010) and, secondly, by the fact that clofazimine is often used in combination with other antimycobacterial agents known to possess cardiotoxic potential, specifically bedaquiline and delaminid (Tadolini et al., 2016; Wallis, 2016).

Importantly, however, little is known about the effects of clofazimine on the reactivity of human platelets, cells which are not only key mediators of thrombosis, but which are also major drivers of neutrophil-mediated inflammation, largely mediated via upregulated expression of the adhesion molecule, CD62P (P-selectin), stored in cytoplasmic $\alpha$-granules (Wagner, 2005). In addition to pro-thrombotic activity, platelets also possess pro-arrhythmogenic potential (de Jong and Dekker, 2010). Accordingly, the current study has been undertaken with the primary objective of investigating the effects of clofazimine, as well as those of the first-line anti-TB drugs, isoniazid and rifampicin, on expression of the key mediator, CD62P, by agonist-activated platelets in vitro, as well as the modulatory potential of the membrane-stabilizing agent, $\alpha$-tocopherol.

\section{MATERIALS AND METHODS}

\section{Ethics Committee Approval}

Permission to undertake this study and draw blood from healthy, adult human volunteers (mean age 32.2 years; 26 females: 16 males) was granted by the Research Ethics Committee of the Faculty of Health Sciences, University of Pretoria in full compliance with the World Medical Association Declaration of Helsinki 2013 (Approval No. 116/2017). Prior written informed consent was obtained from all blood donors each of whom underwent a health check (including measurement of blood pressure) by an experienced, qualified nursing sister prior to the blood draw.

\section{Antibiotics and Other Pharmacologic Agents}

Clofazimine, isoniazid, rifampicin, and $\alpha$-tocopherol (vitamin E), were purchased from the Sigma Chemical Co. (St Louis, MO, United States). All of these agents were dissolved to stock concentrations in dimethylsulphoxide (DMSO), with appropriate solvent controls included in the assays of platelet activation described below.

Unless indicated, all other chemicals were also purchased from the Sigma Chemical Co.

\section{Platelet-Rich Plasma (PRP) and Buffy Coat Preparation}

To prepare PRP, blood (anti-coagulated with five units of preservative-free heparin/mL blood) was centrifuged at $250 \times g$ for $10 \mathrm{~min}$ at room temperature within $15 \mathrm{~min}$ of venepuncture as described previously ( $\mathrm{Nel}$ et al., 2016). The essentially erythrocyte- and leukocyte-free upper layer of PRP was decanted and used in the experiments described below. Buffy coat suspensions prepared by sedimentation of heparinised blood at $37^{\circ} \mathrm{C}$ were used for analysis of neutrophil;platelet (NP) heterotypic aggregate formation (Nel et al., 2017).

\section{Effects of the Test Agents on Platelet Activation}

Platelet-rich plasma $(20 \mu \mathrm{L})$ was added to $980 \mu \mathrm{L}$ of Hanks' balanced solution (HBSS, indicator-free, $1.25 \mathrm{mM}$ calcium, $\mathrm{pH}$ 7.4) and incubated for $5 \mathrm{~min}$ at $37^{\circ} \mathrm{C}$ followed by addition of $1-2 \mu \mathrm{L}$ clofazimine $(0.625-10 \mathrm{mg} / \mathrm{L}$, final), isoniazid or rifampicin (5 and $10 \mathrm{mg} / \mathrm{L}$, final), or DMSO to control systems $(0.1-0.2 \%$, final), and the test tubes incubated for a further period of $10 \mathrm{~min}$ at $37^{\circ} \mathrm{C}$. Thereafter, the potent, primary activator of platelets, thrombin (from human plasma, used at a final sub-maximal concentration of $0.31 \mathrm{NIH}$ units $/ \mathrm{mL}$ ), or the secondary, autocrine activator, adenosine $5^{\prime}$-diphosphate (ADP, $100 \mu \mathrm{M}$, final maximal concentration), agonists of the platelet proteinase-activated receptors (PARs) 1 and 4 and the purinergic P2Y1 and P2Y12 receptors, respectively, or an equal volume of HBSS (background to detect spontaneous platelet activation), were added to the tubes. These were then incubated for a further period of $5 \mathrm{~min}$ at $37^{\circ} \mathrm{C}$ and processed immediately thereafter for analysis by flow cytometry. In an additional series of experiments limited to clofazimine-treated systems, the effects of pre-treatment of PRP with $\alpha$-tocopherol $(30 \mathrm{mg} / \mathrm{L}$, final) on clofazimine $(0.625$ and $1.25 \mathrm{mg} / \mathrm{L})$-mediated potentiation of ADP-induced platelet activation were investigated.

\section{Measurement of Platelet Activation}

Platelet activation was measured by flow cytometry as the proportion (\%) of $\mathrm{CD}_{2} 2 \mathrm{a}^{+}$platelets expressing the $\alpha$-granulederived adhesion molecule, CD62P, considered to be a key mediator of platelet activation and pro-thrombotic activity (Wagner, 2005). Following incubation with the various antimicrobial agents, the platelet suspensions (PRP) were stained with $5 \mu$ l each of a murine anti-human platelet CD42aphycoerythrin (PE)-labeled monoclonal antibody and an 
anti-human CD62P-fluorescein isothiocyanate (FITC)-labeled monoclonal antibody (both from Becton Dickenson, San Jose, CA, United States) to detect the total and activated platelet populations, respectively. After $15 \mathrm{~min}$ of incubation in the dark, the samples were analyzed on a Gallios flow cytometer (Beckman Coulter, Miami, FL, United States) and the results expressed as the percentage of activated platelets with 50,000 cells interrogated during each measurement. The gating and analytical strategies used in a typical experiment are shown in the Supplementary Figure S1.

\section{Neutrophil:Platelet (NP) Aggregate Formation}

In this more limited series of experiments, the effects of clofazimine only at fixed concentrations of 2,5 and $5 \mathrm{mg} / \mathrm{L}$ on NP interactions were investigated. Buffy coat $(20 \mu \mathrm{L})$, suspended in $980 \mu \mathrm{L}$ HBSS, was processed as above for PRP and NP aggregate formation measured following activation with thrombin (0.31 $\mathrm{NIH}$ units/mL) or ADP $(100 \mu \mathrm{M})$ as described previously (Nel et al., 2017). Following incubation, the cells were stained for $15 \mathrm{~min}$ at room temperature in the dark with a cocktail consisting of $5 \mu \mathrm{L}$ of each of the following, murine, antihuman, fluorochrome-labeled monoclonal antibodies: CD16allophyocyanin (Biolegend, San Diego, CA, United States), CD42a-PE (Becton Dickenson) and CD45-Krome Orange (Beckman Coulter) to enable detection of neutrophils, platelets and total leukocytes, respectively. This was followed by analysis of the cell suspensions at a slow flow rate using the Gallios flow cytometer. NP heterotypic aggregate formation was determined as $\mathrm{CD} 16^{+} / \mathrm{CD} 45^{+}$neutrophils co-expressing CD42a. Results are expressed as the relative median fluorescence intensity (MFI) of $\mathrm{CD} 42 \mathrm{a}$ as emitted by $\mathrm{CD} 16^{+} / \mathrm{CD} 45^{+}$neutrophils as an index of the magnitude of the interaction of platelets with individual neutrophils.

\section{Expression and Statistical Analysis of Results}

The results of each series of experiments are expressed as the mean values \pm standard deviations (SDs) with the numbers of different donors and replicate experiments clearly indicated. Statistical analysis was performed using WinStat statistical software with levels of statistical significance calculated using the Mann-Whitney $U$-test for comparison of non-parametric data. A $P$-value of $\leq 0.05$ was considered significant.

\section{RESULTS}

\section{Platelet and Leukocyte Counts}

The mean numbers of platelets and total leukocytes in the PRP preparations were $262 \pm 85 \times 10^{9} / \mathrm{L}$ and $0.15 \pm 0.1 \times 10^{9} / \mathrm{L}$, respectively, while the numbers of platelets and neutrophils in the buffy coat suspensions were $468 \pm 219 \times 10^{9} / \mathrm{L}$ and $4.6 \pm 2.2 \times 10^{9} / \mathrm{L}$, respectively, (mean values \pm SDs).

\section{Effects of the Test Antimicrobial Agents on Spontaneous and ADP- or Thrombin-Activated Expression of Platelet CD62P}

Of the 3 agents tested, only clofazimine was found to cause a statistically significant augmentation of both ADPand thrombin-activated expression of CD62P by platelets as shown in Figure 1A. Clofazimine-mediated augmentation of CD62P expression attained statistical significance at threshold concentrations of 0.625 and $2.5 \mathrm{mg} / \mathrm{L}$ for ADP- and thrombinactivated systems, respectively. With respect to the effects of clofazimine alone (in the absence of ADP or thrombin), slight

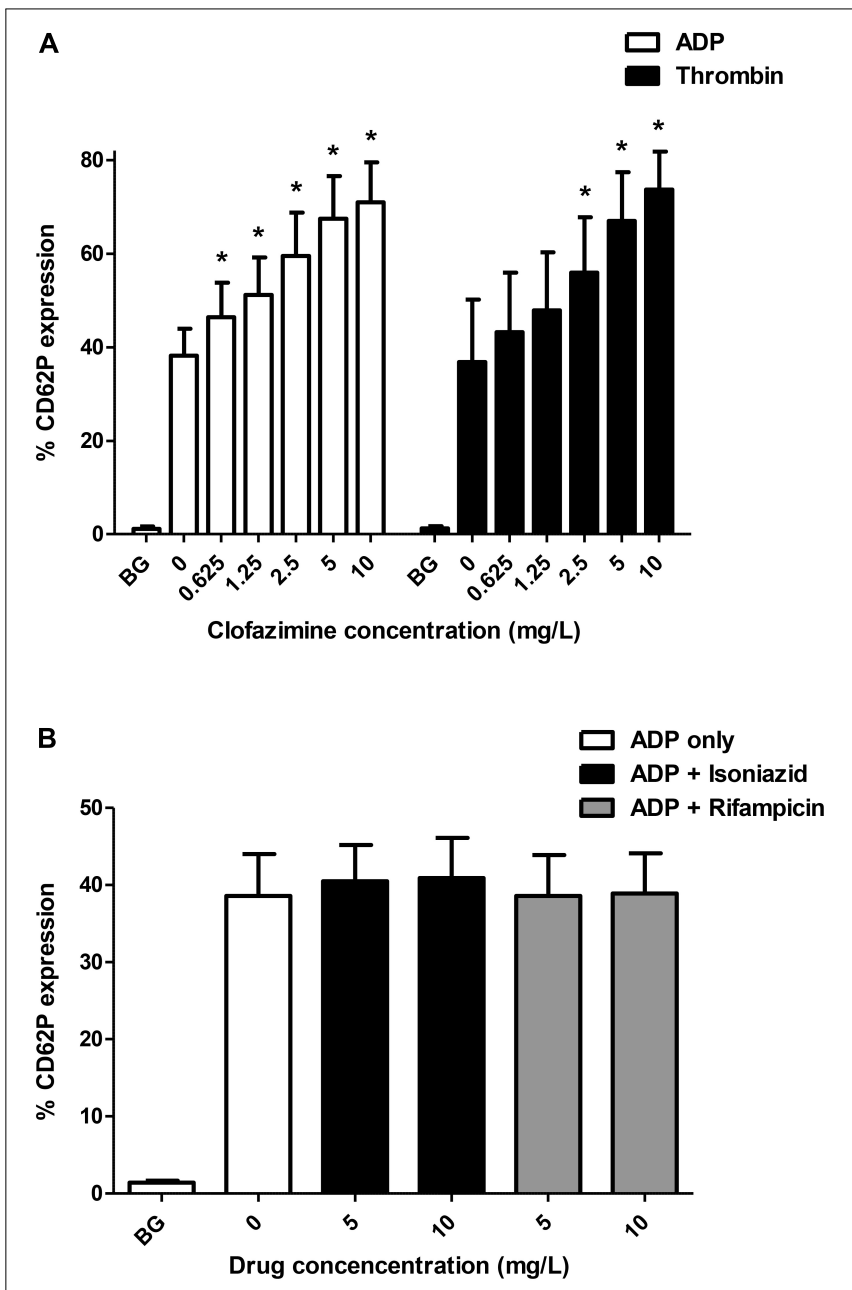

FIGURE 1 | (A) Effects of clofazimine (0.625-10 mg/L) on CD62P expression on unstimulated (BG, background) platelets and cells activated with ADP (100 $\mu \mathrm{M}$; data from 11 experiments using PRP from seven different donors) or thrombin $(0.31 \mathrm{NIH}$ units $/ \mathrm{mL}$; data from eight experiments using PRP from five different donors). The results are expressed as the $\%$ activated platelets. The effects of isoniazid and rifampicin at concentrations of 5 and $10 \mathrm{mg} / \mathrm{L}$ on ADP $(100 \mu \mathrm{M})$-activated expression of CD62P on platelets are shown in Panel (B) (data from five separate experiments). ${ }^{*} P<0.05$ for comparison of clofazimine-treated ADP- or thrombin-activated systems with the corresponding clofazimine-free control systems. 
augmentation of expression of CD62P was observed at 5 and $10 \mathrm{mg} / \mathrm{L}$ of this agent, the mean $( \pm \mathrm{SD})$ values for the untreated control system and systems treated with 5 and $10 \mathrm{mg} / \mathrm{L}$ clofazimine being $1.09 \pm 0.47 \%, 1.35 \pm 0.63 \%$ and $1.48 \pm 0.56 \%$, respectively, (data from 6 experiments using PRP from 4 different donors).

Pre-treatment of PRP with $\alpha$-tocopherol (30 mg/L) caused partial, albeit statistically significant, attenuation of clofaziminemediated augmentation of ADP-activated expression of platelet CD62P, decreasing the mean $( \pm$ SD) levels of expression of CD62P from $44.3 \pm 8.0$ to $39.3 \pm 7.2 \%(P<0.05)$ and from $49.4 \pm 8.1$ to $42.2 \pm 8.7 \%(P<0.05)$ for systems treated with 0.625 and $1.25 \mathrm{mg} / \mathrm{L}$ clofazimine, respectively. The corresponding values for the clofazimine-free systems in the absence and presence of $\alpha$-tocopherol were $35.0 \pm 6.7 \%$ and $35.0 \pm 7.1 \%$, respectively, while the magnitude of CD62P expression on unstimulated platelets was $1.2 \pm 0.4 \%$ (data from 8 separate experiments using PRP from 6 different donors).

With respect to isoniazid and rifampicin, the results for the unstimulated, and ADP-activated control system and systems treated with 5 and $10 \mathrm{mg} / \mathrm{L}$ of isoniazid are shown in Figure 1B, demonstrating lack of activity of these agents. Likewise, none of the test agents affected either spontaneous or thrombin-activated expression of CD62P (not shown).

\section{NP Aggregation}

The effects of clofazimine on ADP- and thrombin-activated NP aggregation formation are shown in Figure 2. At both concentrations tested $(2.5$ and $5 \mathrm{mg} / \mathrm{L})$, clofazimine caused significant potentiation of ADP-formation of NP aggregates. Although similar trends were observed with thrombin-activated

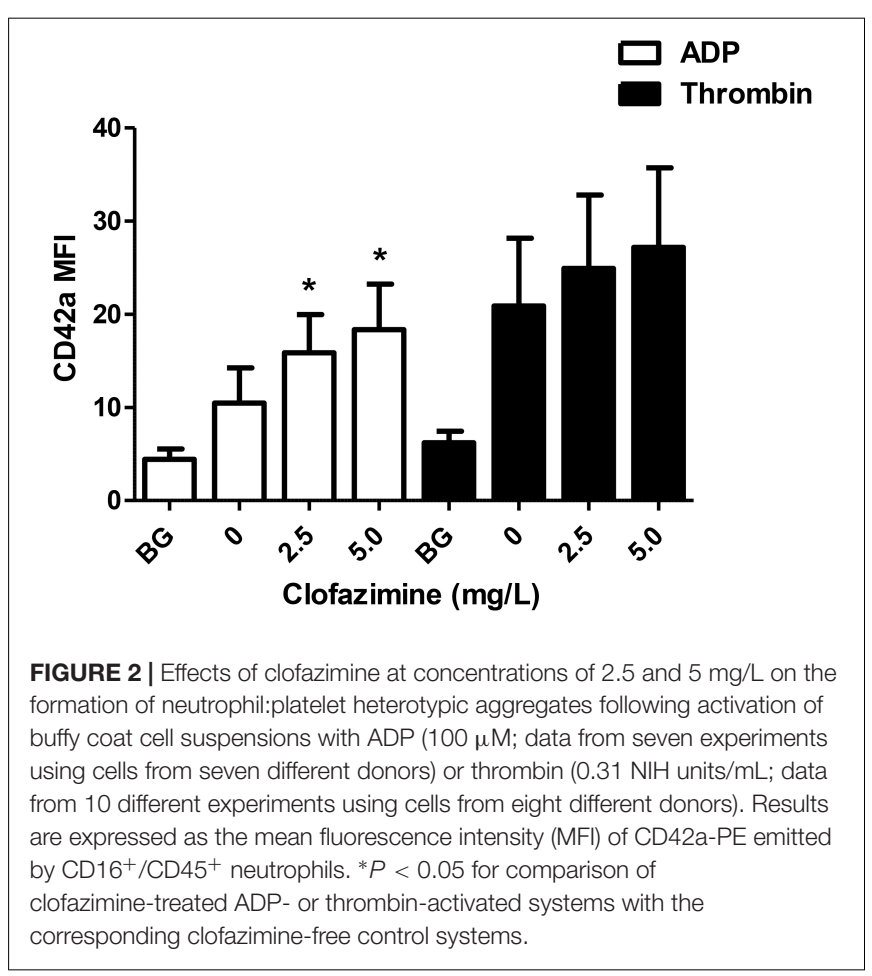

systems, the differences between the control and clofaziminetreated systems did not achieve statistical significance.

\section{DISCUSSION}

The findings of the current study demonstrate that clofazimine, but not isoniazid or rifampicin, potentiates ADP- and thrombinmediated activation of human platelets in vitro, measured according to the magnitude of upregulation of expression of the $\alpha$-granule-derived adhesion molecule, CD62P, as well as ADPactivated formation of NP heterotypic aggregates. In this context, $\mathrm{CD} 62 \mathrm{P}$ is widely recognized as being a key player in plateletdriven, systemic activation of neutrophils, as well as monocytes and vascular endothelium, effectively bridging thrombosis and inflammation (Wagner, 2005; Lisman, 2018). Importantly, the concentrations of clofazimine at which augmentation of platelet activation was observed are close to those reported to occur in serum following administration of this agent, ranging from 0.23 to $1.4 \mathrm{mg} / \mathrm{L}$ depending on dose and frequency of administration (Yawalkar and Vischer, 1979; Diacon et al., 2015). However, due to its lipophilicity ( $\mathrm{pKa}=8,51$ ) and unusual pharmacokinetics, cell and tissue concentrations of clofazimine are considerably higher (Yawalkar and Vischer, 1979).

Although the mechanism of clofazimine-mediated augmentation of platelet activation remains to be established, it is nevertheless likely to be membrane-targeted and somewhat nonspecific. This contention is based on the lipophilicity and cationic amphiphilic properties of clofazimine. These promote binding of cationic amphiphilic drugs to integral anionic membrane phospholipids, which anchor and maintain the functions of key membrane proteins in both eukaryotic and prokaryotic cells (Abraham et al., 2007; Nussio et al., 2007; Cholo et al., 2017). These events, in turn, lead to membrane destabilization, altered conformation of membrane proteins and loss of function, which, in the case of ion transporters, results in dissipation of the membrane potential and membrane depolarization. This scenario is supported by the observation that pre-treatment of platelets with $\alpha$-tocopherol, an agent which attenuates the binding of cationic amphiphiles to phospholipids (Scuntaro et al., 1996), caused partial attenuation of clofazimine-mediated hyperreactivity of these cells. In this context, it is noteworthy that membrane depolarization has been reported to enhance receptor-mediated activation of platelets (Palés et al., 1988).

\section{CONCLUSION}

Irrespective of putative mechanisms of action, the findings of the current study have identified a potentially adverse, prothrombotic activity of clofazimine, which, although unexplored in the clinical setting, may predispose patients with MDR/XDRTB for development of cardiac dysfunction. This contention is of particular relevance in the context of possible synergistic cardiotoxicity resulting from multidrug therapy with clofazimine combined with agents such as bedaquiline and delaminid.This situation may be further exacerbated by the co-existent use of 
pro-atherosclerotic, anti-retroviral agents in settings with a high prevalence of HIV infection. Insights into mechanisms of these chemotherapy-related adverse events may provide a basis for future exploration of platelet-targeted therapies.

\section{AUTHOR CONTRIBUTIONS}

RA, AT, JN, CD, and GR were involved in performance of the various laboratory investigations. All authors contributed to the design of the study, analysis and interpretation of the data, and compilation of the manuscript.

\section{REFERENCES}

Abraham, T., Marwaha, S., Kobewka, D. M., Lewis, R. N., Prenner, E. J., Hodges, R. S., et al. (2007). The relationship between the binding to and permeabilization of phospholipid bilayer membranes by GS14dfK4, a designed analog of the antimicrobial peptide gramicidin S. Biochim. Biophys. Acta 1768, 2089-2098. doi: 10.1016/j.bbamem.2007.06.023

Aung, K. J., Van Deun, A., Declercq, E., Sarker, M. R., Das, P. K., Hossain, M. A., et al. (2014). Successful '9-month Bangladesh regimen' for multidrug-resistant tuberculosis among over 500 consecutive patients. Int. J. Tuberc. Lung Dis. 18, 1180-1187. doi: 10.5588/ijtld. 14.0100

Cholo, M. C., Mothiba, M. T., Fourie, B., and Anderson, R. (2017). Mechanisms of action and therapeutic efficacies of the lipophilic antimycobacterial agents clofazimine and bedaquiline. J. Antimicrob. Chemother. 72, 338-353. doi: 10. $1093 / j a c / d k w 426$

Choudhri, S. H., Harris, L., Butany, J. W., and Keystone, J. S. (1995). Clofazimine induced cardiotoxicity - a case report. Lepr. Rev. 66, 63-68. doi: 10.5935/03057518.19950009

Chung, W. S., Lin, C. L., Hung, C. T., Chu, Y. H., Sung, F. C., Kao, C. H., et al. (2014). Tuberculosis increases the subsequent risk of acute coronary syndrome: a nationwide population-based cohort study. Int. J. Tuberc. Lung Dis. 18, 79-83. doi: 10.5588/ijtld.13.0288

de Jong, J. S., and Dekker, L. R. (2010). Platelets and cardiac arrhythmia. Front. Physiol. 1:166. doi: 10.3389/fphys.2010.00166

Diacon, A. H., Dawson, R., von Groote-Bidlingmaier, F., Symons, G., Venter, A., Donald, P. R., et al. (2015). Bactericidal activity of pyrazinamide and clofazimine alone and in combinations with pretomanid and bedaquiline. Am. J. Respir. Crit. Care Med. 191, 943-953. doi: 10.1164/rccm.201410-1801OC

Huaman, M. A., Kryscio, R. J., Fichtenbaum, C. J., Henson, D., Salt, E., Sterling, T. R., et al. (2017). Tuberculosis and risk of acute myocardial infarction: a propensity score-matched analysis. Epidemiol. Infect. 145, 1363-1367. doi: 10. 1017/S0950268817000279

Li, S., Chan, J. Y., Li, Y., Bardelang, D., Zheng, J., Yew, W. W., et al. (2016). Complexation of clofazimine by macrocyclic cucurbit[7]uril reduced its cardiotoxicity without affecting the antimycobacterial efficacy. Org. Biomol. Chem. 14, 7563-7569. doi: 10.1039/c6ob01060a

Lisman, T. (2018). Platelet-neutrophil interactions as drivers of inflammatory and thrombotic disease. Cell Tissue Res. 371, 567-576. doi: 10.1007/s00441-0172727-4

Nel, J. G., Durandt, C., Mitchell, T. J., Feldman, C., Anderson, R., and Tintinger, G. R. (2016). Pneumolysin mediates platelet activation in vitro. Lung 194, 589-593. doi: 10.1007/s00408-016-9900-5

Nel, J. G., Durandt, C., Theron, A. J., Tintinger, G. R., Pool, R., Richards, G. A., et al. (2017). Pneumolysin mediates heterotypic aggregation of neutrophils and platelets in vitro. J. Infect. 74, 599-608. doi: 10.1016/j.jinf.2017.02.010

\section{FUNDING}

This study was supported by The National Research Foundation of South Africa (CF, ongoing support) and The National Health Laboratory Service of South Africa Research Trust (MC, Grant 004 94648).

\section{SUPPLEMENTARY MATERIAL}

The Supplementary Material for this article can be found online at: https://www.frontiersin.org/articles/10.3389/fphar. 2018.01335/full\#supplementary-material

Nussio, M. R., Sykes, M. J., Miners, J. O., and Shapter, J. G. (2007). Characterisation of the binding of cationic amphiphilic drugs to phospholipid bilayers using surface plasmon resonance. ChemMedChem 2, 366-373. doi: 10.1002/cmdc. 200600252

Palés, J., López, A., and Gual, A. (1988). Platelet membrane potential as a modulator of aggregating mechanisms. Biochim. Biophys. Acta 944, 85-89. doi: 10.1016/ 0005-2736(88)90319-7

Pepper, D. J., Marais, S., Wilkinson, R. J., Bhaijee, F., Maartens, G., McIlleron, H., et al. (2010). Clinical deterioration during antituberculosis treatment in Africa: incidence, causes and risk factors. BMC Infect. Dis. 10:83. doi: 10.1186/14712334- 10-83

Scuntaro, I., Kientsch, U., Wiesmann, U. N., and Honegger, U. E. (1996). Inhibition by vitamin $\mathrm{E}$ of drug accumulation and of phospholipidosis induced by desipramine and other cationic amphiphilic drugs in human cultured cells. Br. J. Pharmacol. 119, 829-834. doi: 10.1111/j.1476-5381.1996.tb15 747.x

Tang, S., Yao, L., Hao, X., Liu, Y., Zeng, L., Liu, G., et al. (2015). Clofazimine for the treatment of multidrug-resistant tuberculosis: prospective, multicentre, randomized controlled study in China. Clin. Infect. Dis. 60, 1361-1367. doi: $10.1093 / \mathrm{cid} / \mathrm{civ} 027$

Trébucq, A., Schwoebel, V., Kashongwe, Z., Bakayoko, A., Kuaban, C., Noeske, J., et al. (2018). Treatment outcome with a short multidrug-resistant tuberculosis regimen in nine African countries. Int. J. Tuberc. Lung Dis. 22, 17-25. doi: 10.5588/ijtld.17.0498

Wagner, D. D. (2005). New links between inflammation and thrombosis. Arterioscler. Thromb. Vasc. Biol. 25, 1321-1324. doi: 10.1161/01.ATV. 0000166521.90532 .44

Wallis, R. S. (2016). Cardiac safety of extensively drug-resistant tuberculosis regimens including bedaquiline, delamanid and clofazimine. Eur. Respir. J. 48, 1526-1527. doi: 10.1183/13993003.012072016

Yawalkar, S. J., and Vischer, W. (1979). Lamprene (clofazimine) in leprosy. Lepr. Rev. 50, 135-144. doi: 10.5935/0305-7518.19790020

Conflict of Interest Statement: The authors declare that the research was conducted in the absence of any commercial or financial relationships that could be construed as a potential conflict of interest.

Copyright (C) 2018 Anderson, Theron, Nel, Durandt, Cholo, Feldman and Tintinger. This is an open-access article distributed under the terms of the Creative Commons Attribution License (CC BY). The use, distribution or reproduction in other forums is permitted, provided the original author(s) and the copyright owner(s) are credited and that the original publication in this journal is cited, in accordance with accepted academic practice. No use, distribution or reproduction is permitted which does not comply with these terms. 